\title{
An Ischemia Detector Based on Wavelet Analysis of Electrocardiogram ST Segments
}

\author{
FJR Sales $^{1}$, S Jayanthi $^{1,2}$, SS Furuie $^{1}$, RKH Galvão ${ }^{2}$ \\ ${ }^{1}$ Heart Institute(Incor) - University of São Paulo Medical School, São Paulo, SP, Brazil \\ ${ }^{2}$ Instituto Tecnológico de Aeronautica (ITA), São José dos Campos, SP, Brazil
}

\begin{abstract}
This paper analyses a strategy for ischemia detection based on wavelet decomposition of the ST segment. The wavelet transform is used as a pre-processing tool for linear discriminant classifier. In order to minimize generalization problems caused by correlations between the classification variables, a selection algorithm is employed to choose a subset of wavelet coefficients with appropriate discriminability and small collinearity.

When applied to a set with small morfologic variability, good results are obtained: $98.5 \%$ of accuracy and a ROC Area equal to 0.98. However, when the training set has a high within-class scatter, the discriminant model yields poor results.
\end{abstract}

\section{Introduction}

The influence of myocardial ischemia in eletrocardiograms is well known. The deficiency of oxygen-carrying blood in ventricular cells due to coronary arteries blockage causes an odd behaviour in the repolarization of the muscular tissue in the ventricule. This process appears as an alteration in the $\mathrm{T}$ wave of an eletrocardiogram. The analysis of $\mathrm{T}$ wave is the main method of detecting ischemia in patients. [1]

Detection of ischemia in early stages is difficult because this pathology does not have clear symptoms such that patient complains can be easily identified. The only clear signals are an eventual angina or a heart infarct, when it is late to start a preventive treatment.

One exam that has potential in detecting heart ischemia in early stages is the Holter exam, because of its long duration. The task of analysing the whole exam results is tiresome and tedious and pathological episodes might be overseen by the pratictioner. An automatic analyser in long duration electrocardiograms is therefore useful to detect in early stages ischemic episodes in patients that might be apparently healthy.

The method used to identify ischemic from nonischemic episodes that we propose makes intensive use of the wavelet transform, due to its capacity of extracting information from the signal shape without loss of important time information.

All this processing was done over the Long Term ST Database located at www.physionet.org.

\section{Methods}

The block diagram below shows the steps taken to build our ischemia detector. The individual steps are described separately in the following topics.

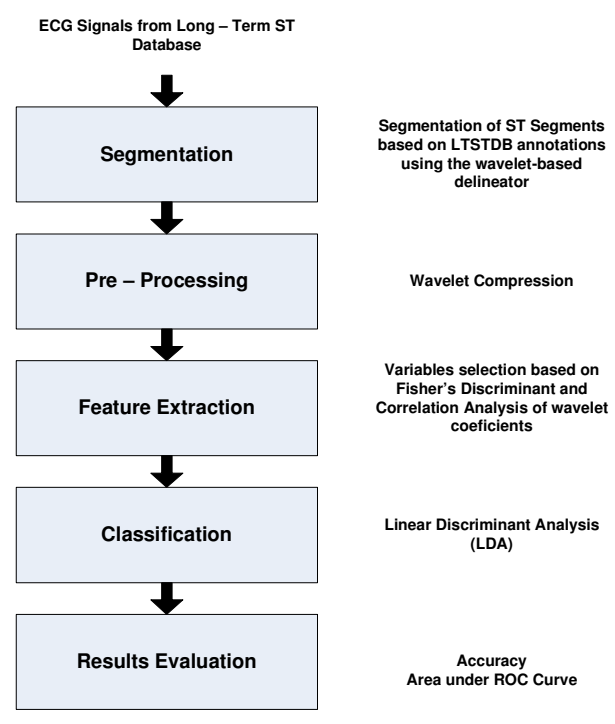

Figure 1: Block diagram of main stages of this work

\section{Long -Term ST Database}

To develop this work, signals from Long - Term ST Database (LTSTDB) [8] have been used. From the 43 registers which are freely available in Physionet, only two subsets were used, more specifically, those signals with ML2 and 0 leads. We created two data scenarios, one with small number of patients (ML2 subgroup) and the other with greater number of cases (0-lead subgroup). 


\section{Segmentation}

To identify the ST segments we used the same approach of [3]. The idea is to use smoothing properties of the wavelet transform when the mother wavelet is the derivative of a smoothing function [3]. Due to that, the zero crossings of a wavelet transform is related to local maxima or minima and local maxima or minima of the wavelet transform is related to maximum slopes of the original signal[3].

The prototype wavelet used was the quadratic spline Fourier Transform as in [2]

$$
\Psi(j w)=j w\left[\frac{\sin (w / 4)}{w / 4}\right]^{4}
$$

This prototype wavelet is the derivative of the convolution of four rectangular pulses, which is a low pass function, satisfying therefore the properties stated before. Considering the sampling frequency of $250 \mathrm{~Hz}$ of the database used, the continuous wavelet transform using the mother wavelet shown above is equivalent to use the set of Finite Impulse Response (FIR) filters in the Mallat's algorithm for discrete wavelet transform:

$$
\begin{gathered}
h[n]=\frac{1}{8} *[\delta(n+2)+3 \delta(n+1)+3 \delta(n)+\delta(n-1)] \\
g[n]=2[\delta(n+1)-\delta(n)]
\end{gathered}
$$

$$
\text { Pre - Processing }
$$

Instead of submitting the ST segments directly to the classification phase, they were pre-processed to reduce the number of classifier parameters. Pre-processing was performed by using biorthogonal wavelets, bior1.1 and bior2.2, with decomposition levels from 1 to 5 .

\section{Feature Extraction}

In order to minimise generalisation problems caused by correlation between the classification variables, an algorithm is employed to choose a subset of coefficients with higher discrimination capability and small colinearity. This problem was solved using discriminability concept and the Fisher Discriminant Function [5]. The discriminant power $D_{i}$ of a parameter $x_{i}$ is given by:

$$
D_{i}=\frac{S_{B i}}{S_{W i}}
$$

Where $S_{W i}$ e $S_{B i}$ are within class dispersion and between class dispersion over the parameter $x_{i}$ respectively, that can be determined by:

$$
\begin{gathered}
S_{B i}=\sum_{j=1}^{C} n_{j}\left[m_{i j}-m_{i}\right]^{2} \\
S_{W i}=\sum_{j=1}^{C} s_{i j} \\
S_{i j}=\sum_{k \in I_{j}}\left[x_{i}^{k}-m_{i j}\right]^{2} \\
m_{i j}=\frac{1}{n_{j}} \sum_{k \in I_{j}} x_{i}^{k}
\end{gathered}
$$

Choosing the coefficients using only the Fisher Discriminant might show redundancy of the parameters for separating the classes [5]. Analysis of co-linearity between parameters can improve the capability of obtaining a generalisation of discriminant analysis.

In few words, the method to remove co-linearity was: a) ordering of wavelet coefficients by its Fisher Discriminant; b) for each candidate, check whether the new parameter show correlation with previous coefficients in the set [7] for a defined threshold. The correlation coefficient $r_{i}$ is calculated by the expression

$$
r_{i}=\frac{\sigma\left(\hat{x}_{i}\right)}{\sigma\left(x_{i}\right)}
$$

where $\sigma$ stands for the standard deviation of the set,

$\hat{x}_{i}$ is an estimate of $x_{i}$ through linear regression of the selected parameters. Values of $r_{i}$ close to 1 shows that the inclusion of the evaluated parameter will not bring new information to the model. The threshold used to determine if a parameter should or should not be included is a system parameter.

\section{Classification}

The linear discriminant analysis in this work assumes that there is a gaussian distribution of studied components over each class. This method uses n-dimensional linear surfaces that identifies each object in the n-dimensional space composed by wavelet coefficients [5]. The goal here is to find the surface defined by the function below such that it separates the classes with accuracy. 


$$
\begin{gathered}
f(x)=w^{T} x \\
w^{T}=\left[\begin{array}{llll}
w_{1} & w_{2} & \ldots & w_{n}
\end{array}\right] \\
x=\left[\begin{array}{llll}
x_{1} & x_{2} & \ldots & x_{n}
\end{array}\right]^{T}
\end{gathered}
$$

The $x$ - array contains the wavelet coefficients selected at the Feature Extraction phase and the $w^{T}$-array contains the separation surface coefficients. The linear discriminant analysis determines the threshold to which two classes can be separated with lowest error[5].

\section{Results}

In order to investigate the robustness of the proposed approach, we used two data configuration: a) small number of cases based on ML2 leads; b) larger group based on 0 lead. For first group, that we will call as ML2 subgroup, the traditional method of dividing the patients into three different sets - training, validation and test have become impracticable due to small number of cases. So, a randomly choice of ST segments was done to split them into training and test sets, even using the same register. As we have not a validation set, we adopted a cross-validation technique based on Leave-One-Out method. The used signal sets are shown in the first row of table 1 and the number of cases for training and testing are in table 2. For the second data configuration, which is based on lead 0 (0-subgroup), we considered 11 registers as shown in the second row of table 1 . In this case, we have formed three different sets for classification: training, validation and test as shown in table 3 .

Table 1: Description of registers used in the two data configuration for robustness test

\begin{tabular}{l|l}
\hline Lead & Registers used \\
\hline ML2 & s20011,s20081,s20101,s20121 \\
\hline $\mathbf{0}$ & s20041,s20261,s20281,s20381,s20401,s20441, \\
s20031,s20341,s20361,s20421,s20461
\end{tabular}

Table 2: Number of ST Segments for training and test of the ML2-subgroup

\begin{tabular}{c|c|c}
\hline \multirow{2}{*}{} & \multicolumn{2}{|c}{ Number of ST Segments } \\
\cline { 2 - 3 } & Normal & Ischemia \\
\hline Train & 100 & 200 \\
Test & 75 & 196 \\
\hline
\end{tabular}

Table 3: Registers and number of segments used for training, validation and test of the 0-lead subgroup

\begin{tabular}{c|c|c|c}
\hline \multirow{2}{*}{ Train } & Register & Number of ST Segments \\
\cline { 3 - 4 } & & Normal & Ischemia \\
\hline \multirow{4}{*}{ S20041 } & 69 & 527 \\
& $\mathbf{s 2 0 2 8 1}$ & 244 & 1335 \\
& $\mathbf{s 2 0 3 8 1}$ & 80 & 159 \\
& $\mathbf{s 2 0 4 0 1}$ & 177 & 376 \\
& $\mathbf{s 2 0 4 4 1}$ & 269 & 657 \\
\hline Validation & $\mathbf{s 2 0 2 6 1}$ & 139 & 440 \\
\hline \multirow{4}{*}{ Test } & $\mathbf{s 2 0 0 3 1}$ & 367 & 1575 \\
& $\mathbf{s 2 0 3 4 1}$ & 574 & 2278 \\
& $\mathbf{s 2 0 3 6 1}$ & 0 & 96 \\
& $\mathbf{s 2 0 4 2 1}$ & 449 & 0 \\
& $\mathbf{s 2 0 4 6 1}$ & 452 & 843 \\
\hline & Total & 2820 & 8286 \\
\hline
\end{tabular}

The classification models for both subgroups were obtained using the training sets. Two performance indicators have been calculated for the test sets: accuracy and area under ROC Curve. The results are shown in tables 4 and 5 .

For the ML2-subgroup, the results are good, as shown in table 4. However, the same methodology applied to 0subgroup yields poor results as we can see for the case of Bior2.2 in table 5.

Table 4: Results obtained using ML2-lead signals with bior 1.1 and bior 2.2 wavelets for 1 to 5 decomposition levels using correlation threshold equal to 0,6

\begin{tabular}{c|c|c|c}
\hline \multicolumn{4}{c}{ Wavelet - Bior 1.1 } \\
\hline Level & Average Error (\%) & Accuracy & ROC Area \\
\hline 1 & 3.32 & 0.967 & 0.984 \\
2 & 2,58 & 0,974 & 0.984 \\
3 & 2.58 & 0,971 & 0.985 \\
4 & 3,69 & 0,963 & 0,979 \\
5 & 3,32 & 0,967 & 0,985 \\
\hline \multicolumn{3}{|c}{ Wavelet - Bior 2.2 } \\
\hline Level & Average Error (\%) & Accuracy & ROC Area \\
\hline 1 & 4,06 & 0,959 & 0,980 \\
2 & 4,43 & 0,956 & 0,980 \\
3 & 2,58 & 0,974 & 0,984 \\
4 & 1,48 & 0,985 & 0,983 \\
5 & 4,80 & 0,952 & 0,960 \\
\hline
\end{tabular}


Table 5: Results obtained using 0-lead signals with bior 2.2 wavelet for 1 to 5 decomposition levels using correlation threshold equal a 0.7

\begin{tabular}{c|c|c|c}
\hline \multicolumn{4}{c}{ Wavelet - Bior 2.2 } \\
\hline Level & Average Error (\%) & Accuracy & ROC Area \\
\hline 1 & 44,20 & 0,558 & 0,383 \\
2 & 44,18 & 0,558 & 0,361 \\
3 & 40,90 & 0,591 & 0,394 \\
4 & 43,02 & 0,570 & 0,384 \\
5 & 41,42 & 0,586 & 0,385 \\
\hline
\end{tabular}

\section{Discussion and conclusions}

Observing tables 4 and 5, the obtained results are very different. What should have caused these differences? First of all, in the first case (table 4), the number of signals were smaller than in table 5, and in the building sets, the ST segments were mixed and them randomly chosen. This step probably contaminated both sets, i.e., there was ST segments from the same patients into training and test sets. Another important fact is related to the within-classes scatter. In ML-2 lead signals, the within-classes scatter was smaller than between-classes scatter for some coefficients, which is useful because this is an indicative that the classes are separable. Observing figures 2, 3 and equation (4), this becomes clear.

However, it has not happened to 0-lead signals because of the morphologic differences of ST segments extracted from different patients. The increment in the withinclasses scatter caused a significant reduction on the values of Fisher's Discriminant Function for a large number of coefficients, which implies on a decrement of classifier capability to segregate the classes.

Therefore, an important conclusion to be taken is about the sensibility of this approach to variations on the training patterns. If the variance for most of the coefficients are large, the classifier may have problems at the generalisation phase, causing poor results.

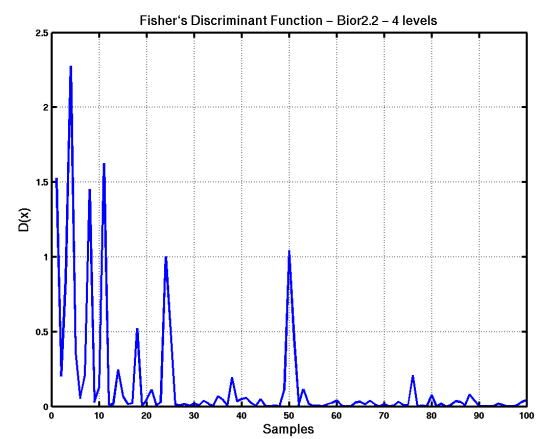

Figure 2: FDF of coefficients from ML2-lead

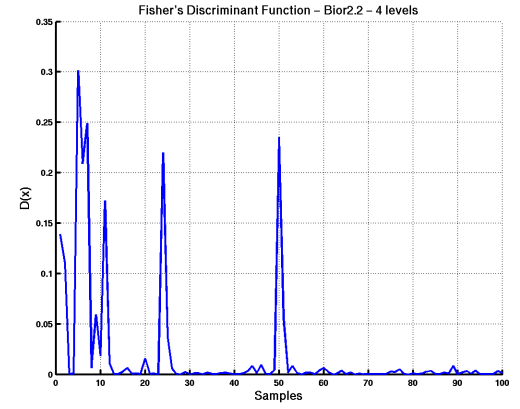

Figure 3: FDF of coefficients from 0-lead

\section{Acknowledgements}

This work was supported by FAPESP (SP, Brazil) grant 04/13205-0 and Fundação Zerbini.

\section{References}

[1] Guyton AC, Hall JE. Textbook of Medical Physiology. $10^{\text {th }}$ ed. Philadelphia: WB Saunders, 2002.

[2] Martínez JP, et al. A Wavelet Based ECG Delineator: Evaluation on Standard Databases, IEEE Trans. on Biom Eng. 2004;51(4):570-581.

[3] Mallat S. Zero Crossings of a Wavelet Transform. IEEE Trans. on Information Theory 1991;37(4):1019-1033.

[4] Shensa MJ. The Discrete Wavelet Transform: Wedding the À Trous and Mallat Algorithms. IEEE Trans. on Signal Processing, 1992;40(10):2464-2482.

[5] Duda O, Hart PE, Stork DG. Pattern Classification. New York: John Wiley, 2002.

[6] Rangayyan RM. Biomedical Signal Analysis: A Case Study Approach. New York: Wiley Interscience, 2002.

[7] Naes T, Mevik BH. Understanding the colinearity problem in regression and discriminant analysis. J.Chemometrics 2001;15:413-426.

[8] Jager F, et al. Long-term ST database: a reference for the development and evaluation of automated ischaemia detectors and for the study of the dynamics of myocardial ischaemia. Medical \& Biological Engineering \& Computing 200341(2):172-183.

Address for correspondence

Name: Fernando Sales

Full postal address: Av. Enéas de Carvalho Aguiar, $44-2^{\text {nd }}$

floor - Serviço de Informática - CEP: 05403-000

E-mail address (optional): fernando.sales@incor.usp.br 